\title{
Fatores que influenciam na decisão da escolha da via de parto: uma revisão
}

\section{integrativa}

\author{
Factors influencing the decision to choose the childbirth mode: an integrative review \\ Factores que influyen en la decisión de elegir el modo de prestación: una revisión integradora
}

Recebido: 21/10/2021 | Revisado: 29/10/2021 | Aceito: 03/11/2021 | Publicado: 06/11/2021

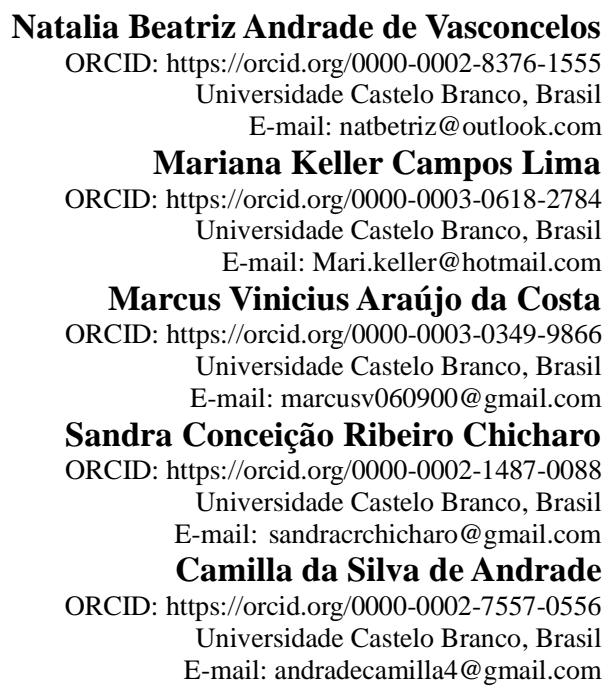

\begin{abstract}
Resumo
Objetivo: Evidenciar a escolha da via de parto sob a perspectiva da autonomia das mulheres. Método: Trata-se de uma revisão integrativa de literatura realizada em Medical Literature Analysis and Retrieval System Online (MEDLINE), Banco de Dados em Enfermagem (BDENF), Literatura Latino Americana e do Caribe em Ciências da Saúde(LILACS) e Scientific Eletronic Library Online (SciELO), com recorte temporal dos últimos 5 anos em português, inglês e espanhol e teve como critério de exclusão artigos incompletos, repetidos nas bases de dados e que fugissem da temática. Resultado e Discussão: A assistência pré-natal é o primeiro passo para o parto e, portanto, é nele que devem ser discutidas todas as dúvidas e medos da gestante. Deve ser ressaltado que o parto é um processo natural e fisiológico que, normalmente, quando bem conduzido, não precisa de condutas intervencionistas. Também devem ser discutidos os valores culturais, sentimentos e as necessidades da gestante. Deve ser informado e orientado à parturiente sobre a evolução do trabalho de parto, lembrando e reconhecendo o papel principal da mulher nesse processo, até mesmo aceitando a sua recusa a condutas que lhe causem constrangimento ou dor. Conclusão: Este estudo procurou apenas realizar uma discussão prévia da importância do conhecimento da gestante sobre os riscos da via de parto a qual serão submetidas e analisar as suas preferências sobre os procedimentos utilizados para o nascimento do bebê, assim nos permitindo coletar informações para legitimar ambas as opções de via de parto.
\end{abstract}

Palavras-chave: Saúde da mulher; Parto normal; Cesárea; Preferência do paciente.

\begin{abstract}
Objective: To demonstrate the choice of the mode of delivery from the perspective of women's autonomy. Method: This is an integrative literature review performed in Medical Literature Analysis and Retrieval System Online (MEDLINE), Database in Nursing (BDENF), Latin American and Caribbean Literature in Health Sciences (LILACS) and Scientific Electronic Library Online (SciELO), with a time frame of the last 5 years in Portuguese, English and Spanish and had as exclusion criteria incomplete articles, repeated in the databases and that escaped the theme. Result and Discussion: Prenatal care is the first step towards childbirth and, therefore, it is in it that all the pregnant women's doubts and fears should be discussed. It should be noted that childbirth is a natural and physiological process that, normally, when properly conducted, does not need interventionist conduct. The pregnant woman's cultural values, feelings and needs should also be discussed. The parturient should be informed and oriented about the evolution of labor, remembering and recognizing the main role of women in this process, even accepting their refusal to conduct that cause them embarrassment or pain. Conclusion: This study only sought to conduct a prior discussion of the importance of knowledge of pregnant women about the risks of the mode of delivery to which they will be submitted
\end{abstract}


and to analyze their preferences about the procedures used for the birth of the baby, thus allowing us to collect information to legitimize both the options for the route of delivery.

Keywords: Women's health; Natural childbirth; Cesarean section; Patient preference.

\begin{abstract}
Resumen
Objetivo: Demostrar la elección del modo de parto desde la perspectiva de la autonomía de las mujeres. Método: Se trata de una revisión integradora de la literatura realizada en el Sistema de Recuperación y Análisis de Literatura Médica en Línea (MEDLINE), Base de Datos en Enfermería (BDENF), Literatura Latinoamericana y del Caribe en Ciencias de la Salud (LILACS) y Biblioteca Electrónica Científica en Línea (SciELO), con un marco temporal de los últimos 5 años en portugués, inglés y español y tenían como criterio de exclusión artículos incompletos, repetidos en las bases de datos y que escapaban a la temática. Resultado y Discusión: La atención prenatal es el primer paso hacia el parto y, por lo tanto, es en él donde se deben discutir todas las dudas y temores de la gestante. Cabe señalar que el parto es un proceso natural y fisiológico que, normalmente, cuando se realiza correctamente, no necesita una conducta intervencionista. También deben discutirse los valores culturales, los sentimientos y las necesidades de la mujer embarazada. La parturienta debe ser informada y orientada sobre la evolución del trabajo de parto, recordando y reconociendo el papel principal de la mujer en este proceso, incluso aceptando su negativa a conductas que le provoquen vergüenza o dolor. Conclusión: Este estudio solo buscó realizar una discusión previa sobre la importancia del conocimiento de las gestantes sobre los riesgos del modo de parto al que serán sometidas y analizar sus preferencias sobre los procedimientos utilizados para el nacimiento del bebé, así permitiéndonos recopilar información para legitimar ambas opciones para la vía de entrega.
\end{abstract}

Palabras clave: Salud de la mujer; Parto normal; Cesárea; Prioridad del paciente.

\title{
1. Introdução
}

Historicamente, o nascimento é denominado como um evento natural, que engloba uma série de significados culturais e sociais, e ambos são fatores que interferem na decisão da escolha da vida de parto (Kottwitz; Gouveia; Gonçalves, 2018). O parto e o nascimento são considerados um marco de grande relevância na vida da mulher e a forma pelo qual esse processo se dá pode ser tanto vivenciado plenamente ou de forma traumática (Rocha; Ferreira, 2020).

Ao longo da história o parto normal era ofertado como a única opção da mulher, excluindo- se qualquer outro tipo de intervenção. Nos dias de hoje, o parto normal é configurado com a imagem de sofrimento e dor. Em razão dessa natureza fisiológica, que exibe a fragilidade da mulher, acaba gerando muitos conflitos emocionais, criando a falsa percepção de que a realização de uma cesárea agendada com antecedência irá proporcioná-la um parto isento de dor (Feitosa et.al, 2017).

No transcorrer do século XX com avanço da ciência, as práticas de assistência ao parto sofreram diversas modificações. A partir daí, ocorreu a transferência do ambiente domiciliar para o hospitalocêntrico, tornando- se um evento dependente de intervenções (Rocha; Ferreira, 2020). Em decorrência da mudança no modelo assistencial e a incidência crescente dos partos programados, a mulher deixou de ser protagonista no processo de parir e no seu direito de escolha (Lima; Freitas, 2020).

O brusco aumento dos números de realização de cesarianas no cenário mundial é a representação de algo ligado à evolução e modernidade da sociedade. Nesse contexto, no Brasil, a cesariana é considerada a cirurgia mais comum realizada em mulheres. A Organização Mundial da Saúde (OMS) recomenda que as taxas de cesáreas fiquem em torno de 10 a $15 \%$ do total (Lima; Freitas, 2020). Ainda assim, de acordo com o modelo de atenção ao parto vigente no país, mais da metade das crianças brasileiras nascem pela via abdominal, esse índice pode chegar até em torno de $80 \%$ nos serviços privados de saúde (Oliveira; Penna, 2018).

A decisão acerca da via de parto pode ser influenciada por uma série de fatores como possíveis complicações, riscos e benefícios (Cassiano et.al, 2021). A preferência da gestante deve ser construída a partir do seu autoconhecimento, das suas expectativas e do acesso às informações durante a gestação. Por isso, torna-se fundamental a aproximação da gestante com o profissional, pois garante uma assistência integral e de qualidade minimizando os seus anseios e dúvidas durante o período da gestação, parto e puerpério (Bacha et al., 2017). 
Desta forma, este estudo tem como objetivo analisar e discutir a via de parto preferida pelas gestantes e identificar os conhecimentos que elas possuem sobre os riscos da via de parto a qual serão submetidas, a partir de revisão integrativa. .

\section{Metodologia}

Trata- se de uma revisão integrativa, de aspecto descritivo, com abordagem qualitativa. Este método de pesquisa permite estabelecer uma síntese e conclusões gerais a respeito de uma área ou assunto específico, realizada de maneira sistemática e ordenada, com o objetivo de contribuir para o conhecimento investigado (Mendes; Silveira; Galvão, 2008).

Para uma revisão integrativa ser construída é necessário desenvolver algumas etapas. Sendo elas: a elaboração da pergunta, coleta bibliográfica, a classificação de dados, a análise e a discussão dos estudos incluídos e os resultados (Crossetti, 2012).

Nesse sentido, foi elaborada a identificação do tema e a seleção da questão de pesquisa que se desenvolveu a partir da temática parto normal e cesáreo. Deste modo, foi delimitada a seguinte questão norteadora: Quais os principais motivos e de qual maneira as gestantes escolhem a via de seu parto?

Realizou - se uma busca nas bases de dados: Medical Analysis and Retrieval System Online (MEDLINE), Banco de dados em Enfermagem (BDENF), Literatura Latino - Americana e do Caribe em Ciências da Saúde (LILACS) e Scientific Eletronic Library Online (SciELO), por meio dos descritores em Ciências da Saúde (DeCS): Saúde da Mulher, Parto Normal, Cesárea e Preferência do paciente. Combinando entre si pelo operador booleano AND. Como critérios de inclusão foram escolhidos artigos disponíveis na íntegra, nos idiomas português, inglês e espanhol, que abordassem o tema entre 2017 e 2021. Os critérios de exclusão foram artigos incompletos, repetidos na base de dados e que não contemplassem o tema abordado.

\section{Resultados e Discussão}

A busca resultou em 678 artigos e, após a aplicação dos critérios de inclusão foram filtrados e selecionados inicialmente o total de 13 artigos. Todavia, 2 encontravam - se duplicados e 3 foram excluídos pelo título, por não abranger a temática.

Assim, foram selecionados para compor a presente revisão integrativa um total de 8 artigos, dos quais (6) apresentam abordagem qualitativa e (2) quantitativa. Logo em seguida, após averiguar as publicações verifica -se que as áreas preponderantes dos artigos encontrados foram (4) saúde pública e (4) enfermagem.

Dentro desse contexto, foi possível observar que a realidade financeira, o medo, a cultura, a influência da sociedade e dos médicos como os principais pilares para as tomadas das decisões de cada gestante. Essas intervenções, não importam de onde venham, terminam por desnaturalizar o parto vaginal e legitimar a via cesárea como a melhor possível em todos os casos e retirar o poder de decisão da mulher (Copelli et al., 2015).

\section{A Escolha da via de Parto}

Foi observado que em algumas narrativas as mulheres relataram que não escolhiam as vias de seu parto e que esse era um procedimento escolhido pelo médico. Essa medicalização tornou o parto um grande evento médico e fez com que a gestante não se sentisse mais ativa no parto de seu filho. O medo da dor também emergiu em muitas literaturas, onde a mulher muitas das vezes se baseava em relatos de parentes e amigos ou até mesmo por influência da mídia por meio de novelas onde o sofrimento e muitos gritos traziam as mulheres o sentimento de medo e horror pelo parto vaginal.

Diversos fatores estão associados a escolha da via de parto envolvendo questões que vão desde a qualidade da assistência obstétrica até às implicações para a saúde da mãe e do bebê, além de se relacionar ao significado do parto atribuído por cada mulher (Weidle., Medeiros., Grave, \& Bosco, 2014). As modalidades de financiamento do parto interferem na 
decisão das gestantes, se não diretamente, pelo menos na formação de uma cultura de procedimentos médicos comuns para cada uma delas. Assim, na rede privada, predominam os partos cesáreos e altos índices de intervenções, muitas vezes desnecessárias, em partos vaginais (Alonso et.al, 2017).

$\mathrm{O}$ advento tecnológico fez com que o parto deixasse o âmbito domiciliar e adentrasse no hospital, processo esse que acometeu não só à assistência obstétrica, mas em toda a área da saúde (Osava, 1997). De acordo com o Sistema de Informações de Nascidos Vivos (SINASC) do Estado do Rio de Janeiro em 2020, a quantidade de partos cesariana foi de 116.308 mil partos contra 82.561 mil partos vaginais. Tal fato demonstra a epidemia de cesáreas que ainda cercam o Brasil.

Em nossa sociedade muitas mulheres que optam por parir a partir da operação cesariana o fazem por imaginarem que esse tipo de parto é indolor e preserva a anatomia vaginal para as relações sexuais (Tedesco et al., 2004). Ultimamente a elevação de partos cesarianas é um fenômeno mundial, sendo o Brasil reconhecido como um dos países com maiores índices e tido como um dos exemplos mais claros de realização deste procedimento mesmo sem indicações (Sakae; Freitas, \& d' Orsi, 2009).

\section{A Conduta informativa do Pré-natal}

A realização do pré-natal representa um papel fundamental em termos de prevenção e/ou detecção precoce de patologias tanto maternas como fetais, permitindo um desenvolvimento saudável do bebê e reduzindo os riscos da gestante, as informações sobre as diferentes vivências devem ser trocadas entre as mulheres e os profissionais de saúde (Brasil, 2000). O pré-natal permite identificar doenças silenciosas como a hipertensão, sífilis, diabetes, pré-eclâmpsia e anemias. O diagnóstico antecipado de algumas dessas doenças permite que o tratamento atinja altas taxas de sucesso para mãe e o bebê. O pré-natal bem acompanhado também permite a identificação de doenças genéticas e de má formação fetal, que se diagnosticadas logo no momento inicial podem ser tratadas intrauterina proporcionando ao recém-nascido uma vida normal (Viellas et al., 2014).

O profissional enfermeiro têm um papel crucial diante das consultas de enfermagem no pré-natal. De acordo com o Ministério da Saúde são necessárias no mínimo 6 consultas durante o período de pré-natal, o enfermeiro irá preparar a mulher para a maternidade, trazendo informações educativas sobre o parto e o cuidado da criança, fazer prevenção, diagnóstico precoce e tratamento de doenças próprias da gestação ou que sejam intercorrências previsíveis dela (Brasil, 2014).

Para a decisão da via de parto é crucial uma maior aproximação do profissional com a gestante, garantindo uma atenção integral e de qualidade, esclarecendo suas dúvidas e anseios no que diz respeito aos aspectos da gestação, parto e puerpério. O papel do enfermeirol na promoção da saúde das mulheres no ciclo gravídico-puerperal, na educação em saúde e na assistência ao processo parir/nascer é um instrumento chave para a construção da autonomia das gestantes (Santana., Lahm., \& Santos, 2015).

A assistência pré-natal é o primeiro passo para o parto e, portanto, é nele que devem ser discutidas todas as dúvidas e medos da gestante. Deve ser ressaltado que o parto é um processo natural e fisiológico que, normalmente, quando bem conduzido, não precisa de condutas intervencionistas. Também devem ser discutidos os valores culturais, sentimentos e as necessidades da gestante. Deve ser informado e orientado à parturiente sobre a evolução do trabalho de parto, lembrando e reconhecendo o papel principal da mulher nesse processo, até mesmo aceitando a sua recusa a condutas que lhe causem constrangimento ou dor. E é claro discutir sobre os tipos de parto e qual é o desejo da mulher naquele momento.

É inegável o papel do profissional que assiste ao parto. Além do conhecimento sobre técnicas de parto, deve ser capacitado a reconhecer que cada mulher é portadora de uma cultura própria, que muitas vezes atribui significados diferentes à vivência do parto. Respeitar essa condição da mulher, orientá-la, acolhê-la em seus questionamentos e dúvidas, ajudá-la, enfim, a fazer dessa experiência um marco em sua trajetória pessoal, são os atributos desejáveis num profissional (Amorim et al., 2012). 


\section{Considerações Finais}

Quando se fala da escolha da via de parto, é possível argumentar sobre os benefícios e malefícios de ambos os procedimentos, tanto o cirúrgico quanto o processo natural. Ao longo desta pesquisa, foi possível observar que o conhecimento da mulher sobre as formas de enfrentar o parto do seu bebê é de suma importância, visto que a escolha do modo do parto resulta de muita influência externa, como a opinião médica, fatores culturais e socioeconômicos.

O processo da maternidade é um fator que inclui um grupo de pessoas além dos pais do bebê, desta forma, existem muitas opiniões, aconselhamentos e uma grande troca cultural entre os pais da criança e um determinado conjunto de pessoas, sejam profissionais ou indivíduos de um círculo social próprio. A influência gerada por outras pessoas tende a ter impacto na decisão da mulher sobre a forma de ter o bebê, levando-a a conviver com o dilema do parto normal ou da cesárea, assim levando a figura feminina a caminhar baseada na opinião de terceiros ao invés da decisão apurada nas suas próprias vontades e conhecimentos.

Após a identificação do tema, foi realizada uma pesquisa de caráter descritivo e com uma abordagem qualitativa, que satisfatoriamente, colaborou para que fossem detectadas as principais influências que contribuem para a escolha da vida de parto por parte da gestante e o seu nível de conhecimento sobre o assunto.

Este estudo procurou apenas realizar uma discussão prévia da importância do conhecimento da gestante sobre os riscos da via de parto a qual serão submetidas e analisar as suas preferências sobre os procedimentos utilizados para o nascimento do bebê, assim nos permitindo coletar informações para legitimar ambas as opções de via de parto.

Sugere-se que estudos futuros possam se interessar por esta temática, aplicando propostas de estender o conhecimento sobre as vias de parto, destacando seus benefícios e malefícios, levando a mulher a conseguir escolher de maneira satisfatória a melhor opção baseada na situação em que se encontra e que fará com que se sinta confortável.

\section{Referências}

Alonso, B. D., da Silva, F. M. B., Latorre, M. R. D. O., Diniz, C. S. G., \& Bick, D. (2017). Caesarean birth rates in public and privately funded hospitals: a cross-sectional study. Revista de Saúde Pública, 51, 101.

Amorim, A. T. C., Araújo, V. K. A., Severiano, R. C. C., \& Davim, R. M. B. (2012). Estratégias utilizadas no processo de humanização ao trabalho de parto: uma revisão. Saúde Coletiva, 9(56), 61-66.

Bacha, A. M., Grassiotto, O. R., Cacique, D. B., Machado, H. C., Carvasan, G. A. F., \& Souza, C. A. B. (2017). Parto normal ou cesárea: a influência do tipo de parto desejado na satisfação materna quanto a assistência hospitalar ao nascimento. Revista de Administração em Saúde, 17, 6.

Brasil. Ministério da Saúde. (2014). Caderneta da Gestante. Brasília - DF. < http://189.28.128.100/dab/docs/portaldab/documentos/caderneta_gestante.pdf >.

Brasil. Ministério da Saúde. (2000). Assistência Pré - natal. Brasília - DF. <bvsms.saude.gov.br/bvs/dicas/90prenatal.html>.

Cassiano, A. N., Menezes, R. J. M. P., Medeiros, S. M., Silva, C. J. A., \& Lima, M. C. R. A. (2021). Atuação do enfermeiro obstétrico na perspectiva das epistemologias do Sul. Escola Anna Nery, 25(1), 1 - 6.

Copelli, F. H. S., Larissa, R., Zampieri, M. F. M., Gregório, V. R. P., \& Custódio, Z. A. O. (2015). Determinants of women's preference for cesarean section. Texto \& Contexto-Enfermagem, 24 (3), 336 - 343.

Crossetti, M. G. O. (2012). Revisão Integrativa de Pesquisa na Enfermagem o rigor científico que lhe é exigido. Revista Gaúcha Enfermagem. 33 (2), 8-9.

Feitosa, R. M. M., Pereira, R. D., Souza, T. J. C. P., Freitas, R. J. M., Cabral, S. A. R., \& Souza, L. F. F. (2017). Fatores que influenciam a escolha do tipo de parto na percepção das puérperas. Revista de Pesquisa Cuidado é Fundamental Online, 9(3),717-726.

Kottwitz, F., Gouveia, H. G., \& Gonçalves, A. C. (2018). Route of birth delivery preferred by mothers and their motivations. Escola Anna Nery, 22(1).

Lima, B. \& Freitas, E. A. M. (2020). A escolha da via de parto: uma revisão integrativa. REFACS, 8(1), 114-125.

Mendes, K. D. S., et al. (2008). Revisão integrativa: método de pesquisa para a incorporação de evidências na saúde e na enfermagem. Texto \& Contexto Enfermagem, 17(4),758-764.

Oliveira V. J., \& Penna, C. M. M. (2018). Every birth is a story: process of choosing the route of delivery. Revista Brasileira de Enfermagem, 71 (3), 1228 1236 
Research, Society and Development, v. 10, n. 14, e386101422112, 2021

(CC BY 4.0) | ISSN 2525-3409 | DOI: http://dx.doi.org/10.33448/rsd-v10i14.22112

Osava, R. H. (1997). Assistência ao Parto no Brasil: O Lugar do Não-Médico (Ana Cristina d'Andreatta Tanaka, orientadora). Tese de Doutorado, São Paulo: Departamento de saúde Materno Infantil, Faculdade de Saúde Pública, Universidade de São Paulo.

Rocha, N. F. F., \& Ferreira, J. (2020). A escolha da via de parto e a autonomia das mulheres no Brasil: uma revisão integrativa. Saúde em Debate, 44(125).

Santana, F. A., Lahm J. V., \& Santos R. P. Fatores que influenciam a gestante na escolha do tipo de parto. Rev Fac Ciênc Méd Sorocaba. $17(3): 123$ - 127.

Sakae, T. M., Freitas, P. F., \& D’orsi, E. (2009). Fatores associados a taxas de cesárea em hospital universitário. Rev Saúde Pública, 43(3):472- 480.

Tedesco, R. P., Filho, N. L. M., Mathias, L., Benez, A. L., Castro, V. C. L., Bourroul, G. M., \& Reis, F. I. (2004). Fatores determinantes para as expectativas de primigestas acerca da via de parto. Revista Brasileira de Ginecologia e Obstetrícia, 26(10).

Viellas, E. F., Domingues, R. M. S. D., Dias, M. A. B., Gama, S. G. N., Filha, M. M. T., Costa, J. N., Bastos, M. H., \& Leal, M. C. (2014). Assistência prénatal no Brasil. Caderno Saúde Púbica, 30(1): 85 - 100.

Weidle, W. G, Medeiros, C. R. G, Grave M. T. Q, \& Bosco, S. M. D. Escolha da via de parto pela mulher: autonomia ou indução? (2014) Cad Saúde Colet., 22 (1): 46-53. 10.1590/1414- 462X201400010008. 